\title{
Molecular cloning and characterization of the family of feline leucine-rich glioma-inactivated (LGI) genes, and mutational analysis in familial spontaneous epileptic cats
}

Yoshihiko Yu*, Daisuke Hasegawa* (D), Aki Fujiwara-Igarashi, Yuji Hamamoto, Shunta Mizoguchi, Takayuki Kuwabara and Michio Fujita

\begin{abstract}
Background: Leucine-rich glioma-inactivated (LGI) proteins play a critical role in synaptic transmission. Dysfunction of these genes and encoded proteins is associated with neurological disorders such as genetic epilepsy or autoimmune limbic encephalitis in animals and human. Familial spontaneous epileptic cats (FSECs) are the only feline strain and animal model of familial temporal lobe epilepsy. The seizure semiology of FSECs comprises recurrent limbic seizures with or without evolution into generalized epileptic seizures, while cats with antibodies against voltage-gated potassium channel complexed/LGI1 show limbic encephalitis and recurrent limbic seizures. However, it remains unclear whether the genetics underlying FSECS are associated with LGI family genes. In the present study, we cloned and characterized the feline LGI1-4 genes and examined their association with FSECs. Conventional PCR techniques were performed for cloning and mutational analysis. Characterization was predicted using bioinformatics software.

Results: The cDNAs of feline LG/1-4 contained 1674-bp, 1650-bp, 1647-bp, and 1617-bp open reading frames, respectively, and encoded proteins comprising 557, 549, 548, and 538 amino acid residues, respectively. The feline LGl1-4 putative protein sequences showed high homology with Homo sapiens, Canis familiaris, Bos taurus, Sus scrofa, and Equus caballus (92\%-100\%). Mutational analysis in 8 FSECs and 8 controls for LGI family genes revealed 3 non-synonymous and 14 synonymous single nucleotide polymorphisms in the coding region. Only one non-synonymous single nucleotide polymorphism in LGI4 was found in 3 out of 8 FSECs. Using three separate computational tools, this mutation was not predicted to be disease causing. No co-segregation of the disease was found with any variant.

Conclusions: We cloned the cDNAs of the four feline LGI genes, analyzed the amino acid sequences, and revealed that epilepsy in FSEC is not a monogenic disorder associated with LGI genes.
\end{abstract}

Keywords: Feline, Epilepsy, Lgi, Cloning and sequence analysis, Molecular characterization

\footnotetext{
* Correspondence: yoshy@shinkei.com; disk-hsgw@nvlu.ac.jp

Department of Clinical Veterinary Medicine, Nippon Veterinary and Life

Science University, 1-7-1 Kyonan-cho, Musashino-shi, Tokyo 180-8602, Japan
} 


\section{Background}

Epilepsy is a chronic functional neurological disorder characterized by recurrent, unprovoked epileptic seizures and a range of loss of consciousness, salivation, muscle spasms, and severe and prolonged convulsion. Epilepsy affects over 50 million people worldwide, and is one of the most common neurological diseases [1]. Although the causes of epilepsy are variable, approximately $70 \%$ is believed to be associated with genetic factors [2]. Thus, understanding the genetic causes is important for developing effective therapeutic strategies for epilepsy [3-5]. Recently, it was also reported that treatment with a chemical corrector, 4-phenylbutyrate ameliorated the increased seizure susceptibility of the LGI1E383A model mouse, a mouse model of familial epilepsy [6].

In 2009, we identified a feline family line with spontaneous recurrent epileptic seizures, and established a colony strain of spontaneous epileptic cats (familial spontaneous epileptic cats [FSECs]) [7]. The FSEC colony consists of large multigenerational pedigrees with multiple cases. FSECs are healthy except for the presence of recurrent epileptic seizures, which have a variable frequency between FSECs. FSECs have two types of clinical seizures, with some FSECs showing both or one seizure pattern(s), while others are asymptomatic. Spontaneous focal limbic seizures with or without evolving into generalized epileptic seizures is one type of seizure observed in FSECs. This seizure type is similar to the historical limbic kindling and/or kainate model of epilepsy [8], as also represents the common seizure type in cats [9-11]. Vestibular stimulation-induced generalized epileptic seizures is the other seizure type in FSECs, and is similar to the widely used EL mouse model, which shows genetic epilepsy [12]. As both the kindling/kainate model and the EL mouse are used to model human mesial temporal lobe epilepsy (MTLE), we consider that FSECs are a natural genetic model of human and feline TLE. We previously reported their clinical, electrophysiological and magnetic resonance imaging findings, which resembled those of human MTLE [13-16]. Further, a familial form of MTLE (FMTLE) was reported in humans [17-20]. Thus, our studies provide support that the FSEC is an animal model of human FMTLE. As such, understanding the genetic causes of FSECs may be useful in exploring the genetics of familial epilepsy.

The leucine-rich glioma-inactivated (LGI) protein family (LGI1-4) play important roles in the development and function of the vertebrate nervous system, including synaptic transmission and myelination. Indeed, dysfunction of these genes and encoded proteins are associated with neurological disorders including genetic epilepsy or autoimmune limbic encephalitis in animals and human [21, 22]. LGI1 has been shown to be causative in the lateral form of familial TLE (also termed autosomal dominant lateral TLE)
[23-25]. In veterinary medicine, feline complex partial seizures with orofacial involvement (FEPSO; also termed feline TLE), characterized by recurrent limbic seizures (e.g., episodic orofacial automatism) with salivation, chewing, licking, and facial twitching, was previously reported [26-28]. Some of those cats have increased antibodies against the voltage-gated potassium channel (VGKC)/ LGI1 complex [27]. Thus, FEPSO is considered to involve, at least in part, VGKC/LGI1 complex antibody-mediated limbic encephalitis (LE), resembling LE in humans.

Interestingly, while FSEC was associated with loss of neurons in the CA3 subarea of the hippocampus [13, 29], the VGKC/LGI1 complex antibody LE in humans is associated with focal CA3 atrophy [30]. A protein-truncating nonsense mutation in the LGI2 gene was identified as a cause of benign familial juvenile epilepsy in the Lagotto Romagnolo dog [31]. Further, LGI4 may contribute to susceptibility to benign familial infantile convulsions and childhood absence epilepsy in humans [32, 33]. Despite these overall findings, there has been no known association of the LGI3 gene with human or veterinary diseases, although LGI3 is highly expressed in the mouse brain in a developmentally- and transcriptionally-regulated manner [34]. As LGI gene family members are highly conserved and have the same overall structure, they may also have similar functions [21], including a predisposition to epilepsy.

In the present study, we examined the hypothesis that the etiology of FSECs is associated with LGI protein family dysfunction [22]. As the cDNA sequences of the feline LGI (fLGI) genes were not previously reported, we first cloned the cDNA of LGI family genes in the Felis catus, predicted the corresponding protein sequence, obtained their basic physical and chemical properties, and then performed phylogenetic and structural analysis. Next, we performed fLGI mutational analysis in FSECs and healthy cats to determine their roles in epilepsy.

\section{Results}

\section{Characterization of full-length feline LGI cDNAs}

We cloned and sequenced the fLGI1, fLGI2, fLGI3, and fLGI4 cDNAs, and sequences were submitted to the DNA Databank of Japan under accession numbers LC309277, LC309278, LC309279, and LC309280, respectively. Nucleotide sequence analysis showed that fLGI1 cDNA contained a 1674-base pair (bp) open reading frame (ORF) encoding a 557-amino acid peptide, fLGI2 cDNA contained a 1650-bp ORF encoding a 549-amino acid peptide, fLGI3 cDNA contained a 1647-bp ORF encoding a 548-amino acid peptide, and fLGI4 cDNA contained a 1617-bp ORF encoding a 538-amino acid peptide.

\section{Amino acid sequence analysis of the feline LGI proteins}

The physicochemical properties of the four LGI proteins were predicted using ProtParam software (Table 1). The 
Table 1 Physiochemical characteristics of the feline leucine-rich glioma-inactivated (LGI) proteins

\begin{tabular}{|c|c|c|c|c|}
\hline Physiochemical characteristics & LGl1 & LGI2 & LGI3 & LGI4 \\
\hline Amino acid & 557 & 549 & 548 & 538 \\
\hline Total number of atoms & 8959 & 8728 & 8683 & 8323 \\
\hline Molecular weight (Da) & $63,817.99$ & $62,249.12$ & $61,887.77$ & $58,979.10$ \\
\hline Isoelectric point & 8.39 & 6.21 & 8.46 & 7.60 \\
\hline $\mathrm{Asp}+\mathrm{Glu}$ & 56 & 63 & 51 & 49 \\
\hline Arg + Lys & 61 & 58 & 56 & 50 \\
\hline Extinction coefficients & $84520^{a} / 83770^{b}$ & $80955^{a} / 80330^{b}$ & $91050^{a} / 90300^{b}$ & $71805^{a} / 70930^{b}$ \\
\hline Aliphatic index & 85.75 & 88.23 & 88.47 & 97.25 \\
\hline Instability index & 42.89 & 53.34 & 44.27 & 47.50 \\
\hline Grand average of hydropathicity & -0.227 & -0.119 & -0.143 & 0.064 \\
\hline
\end{tabular}

${ }^{a}$ Computed value based on the assumption that all cysteine residues appear as half cysteine's; ${ }^{b}$ Assuming that no cysteine appears as a half cysteine

fLGI1, fLGI2, fLGI3, and fLGI4 proteins were predicted to have a signal peptide located at $1-34,1-34,1-30$, and 1-20 amino acids, respectively; three, four, ten, and four O-glycosylation sites, respectively; three, four, three, and one $\mathrm{N}$-glycosylation sites, respectively; $66,57,50$, and 44 phosphorylation sites, respectively; and five, four, five, and five disulfide bonds, respectively (Fig. 1). The subcellular distribution of the fLGI protein was predicted and summarized in Additional file 1.

\section{Prediction of the structures and features of feline LGI proteins}

Secondary structural analysis predicted the proportion of alpha helix, beta sheet, and random coils of the putative fLGI proteins as: fLGI1: $2.15 \%, 30.70 \%$, and $7.15 \%$, respectively; fLGI2: $5.65 \%, 24.95 \%$, and $69.40 \%$, respectively; fLGI3: $3.65 \%, 25.73 \%$, and $70.62 \%$, respectively; and fLGI4: $3.90 \%, 24.90 \%$, and $71.20 \%$, respectively (Fig. 2). Analysis of each deduced amino acid sequence using SMART (Simple Modular Architecture Research Tool) revealed that the leucine-rich repeat domain flanked by the cysteine-rich region (C-terminal) at the $\mathrm{N}$-terminal followed by epitempin (EPTP) domain(s) was common to all fLGI proteins (Fig. 3, Additional file 2). While SMART showed two or three EPTP domains within the LGI4 protein in other mammals, with the exception of Felis catus and Canis familiaris (see Fig. 3), two EPTP domains of fLGI4 positioned in the corresponding regions to other mammals were excluded from the diagram because of E-value thresholds of 0.00075 and 0.0039 . Further, the fLGI family proteins were predicted not to have an interaction in their respective pathways (Additional file 3).

\section{Exonic structure analysis, multiple alignments, and phylogenetic analysis}

The fLGI1-3 genes included eight exons and seven introns, while the fLGI4 gene included nine exons and eight introns. All four genes shared a similar exon structure with those of other mammals (Additional file 4) based on the amino acid and genomic sequences listed in Additional file 5. Homology analysis indicated that the amino acid sequences of the predicted fLGI1-4 proteins were $94.7,92.7,88.5$, and $86.3 \%$ identical, respectively, to the other species listed in Table 2. However, when compared with most typical mammalians including Homo sapiens, Canis familiaris, Bos taurus, Sus scrofa and Equus caballus, the identities were over $92 \%$.

The amino acid sequences of the LGI proteins were aligned with sequences from other species using DNAMAN version 9.122 (Trial version; Lynnon Biosoft, San Ramon, CA, USA) (Additional files 6, 7, 8 and 9). Phylogenetic trees were constructed using MEGA 7.0 (Molecular Evolutionary Genetics Analysis Version 7.0) based on the amino acid sequences of the LGI family listed in Additional file 10 (Fig. 4), and included the proteins identified as homologous to LGI 1-4. The evolutionary history was inferred using the neighbor-joining method. The optimal trees with branch length sums of $0.27374760,0.40249836$, 0.38710421 , and 0.76533180 are shown. The trees are drawn to scale, with branch lengths in the same units as those of the evolutionary distances used to infer the phylogenetic tree. While phylogenetic tree analysis based on the available amino acid sequences revealed that fLGI1, fLGI3, and fLGI4 shared the closest evolutionary relationship with Canis familiaris, fLGI2 showed the closest evolutionary relationship with Sus scrofa and Bos taurus.

\section{Mutational analysis and allele frequency}

Except for exon1 of fLGI2, all exons that contained coding sequences were amplified from the genomic DNA from eight FSECs and eight control cats. It was impossible to design primers for exon 1 of fLGI2 because of its GC rich content. PCR products were compared 


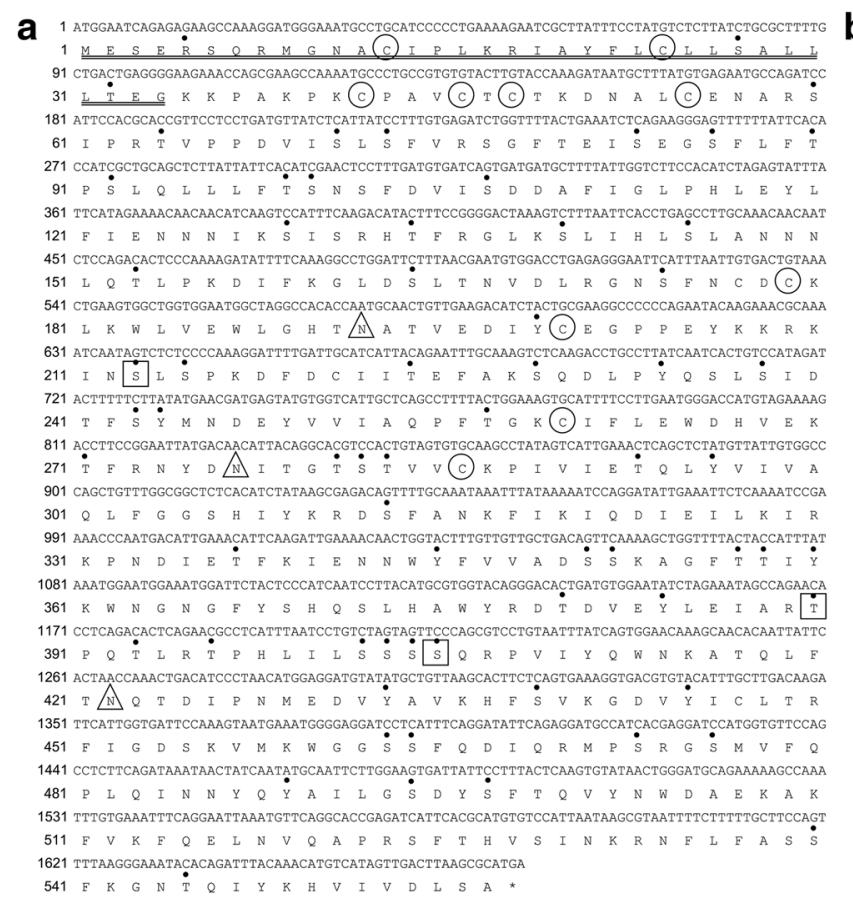

C

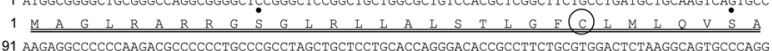

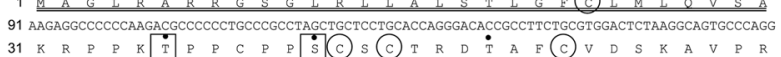

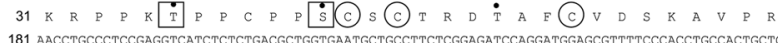
$61 \mathrm{NL}$ P S E V I S L 271 CAGTTCCTGTTGCTCAACTCCAACAAGTTRACTCTGATTGGAGACAATGCCTTCACAGGACTGTCGCACCTGCAGTACCTCTTCATTGAG

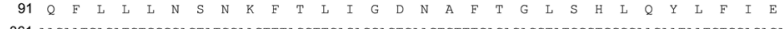

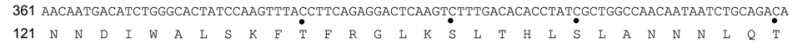

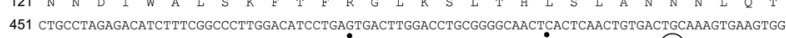
151 I P R D I F R P L D I L S D L D I R G N

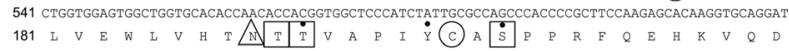

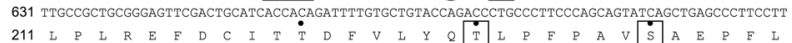

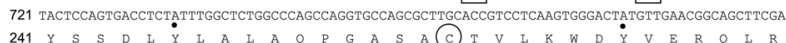
241 Y S S S D L L $\dot{Y}$ L A A L A A Q B G A S A A C T T V L

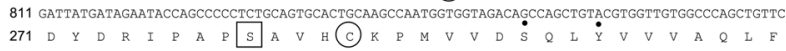

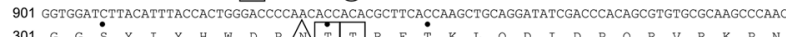
301 G G S S Y I Y Y H 991 GACCTGGAAGCTTTCCGCATTGACGGCGACFGG TACTTTGCCGTGGCCGACAGCTCCAAGGCGGGTGCCACCAGCCPCTACCGCTGGCAC

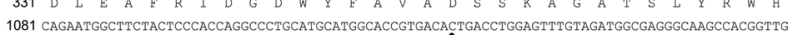
1081 CAGAATGGCTTCTRACTCCCACCAGGCCCTGCATGCATGGCACCGTGACACYGACCTGGAGTTYG TRGATGGCGAGGGCAAGCCACGG TPG 1171 A ATGGTGCTAGTAGCTCCCAGGCACCTGTCATCTAACAGTGGaGCCGCACCCAGAAGCAGTTTGGGGCCCAGGGTGAGGTGACCCAGGTC

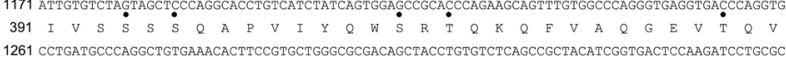

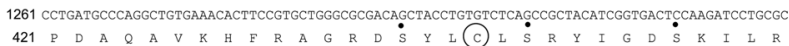
1351 TGGGAGGGCACCCGCTTCTCYTGAAGTGCAGGCCCTGCCCTCCAGGGGCTCGCTGGCCATGCAGCCCTTCCTCGTEGGTGGCCGCCGCTAC

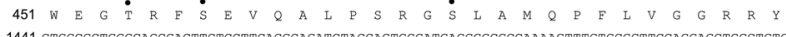

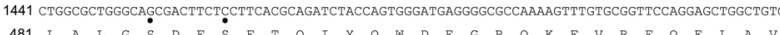

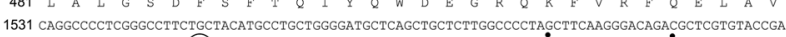

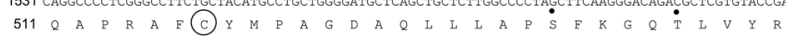

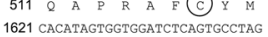

541 H I V V V D

Fig. 1 Nucleotide and deduced amino acid sequences of feline leucine-rich glioma-inactivated (fLGl) genes. a $L G \mid 1$, b fLGl2, c fLGl3, and d fLGl4. Residues are numbered from the $5^{\prime}$ end of the open reading frame (ORF). The putative amino acid sequences are shown by the single-letter amino acid codes. Asterisk indicates the stop codon. The signal peptide sequence is double-underlined. The cysteine residues required for formation of disulfide bonds are indicated with circles. The putative N-linked or O-linked glycosylation sites are indicated with triangles or squares, respectively. The predicted phosphorylation sites are indicated by a dot above the single-letter amino acid code. The sequences reported in this paper have been deposited in the DNA Data Bank of Japan (DDBJ) (LC309277, LC309278, LC309279, and LC309280)

with feline reference genomic sequences (NC_018733.2, NC_018726.2, NC_018726.2, and NC_018737.2). Sequence analysis of the fLGI gene family revealed 41 variants, the majority of which were not in the National Center for b

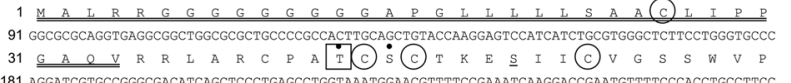

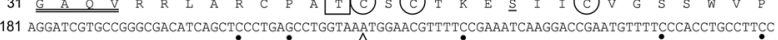

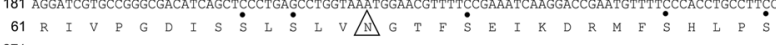
271 CTGCAGCTGCTATTGCTGAATTCTAACTCATTTACAGCCATCCGGGATGATGCTTTTGCTGGACTGTT TCATCTCGAATACCTGTTCAT

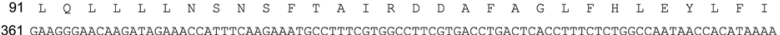
121 E G N 451 GCACTCCCAAGGGATGTCTTCAGTGATTTAGACTCTCTGATTGAACTAGACTTAAGGGGCAATAAATTTGAATGTGACTGCAAAGCCAAG 151 A $\quad$ L $\quad$ P 181 W L $X$ L W L K M T M S T V S D V L C I G P P E 631 GATGTGCCGAGCTTCGACTACGAATGCACAACCACAGATTTTGTCGTTCATCAGACTTTGCCTTACCAGTCGGTTTCAGTGGATACATTC

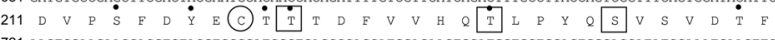

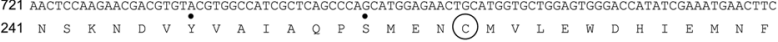

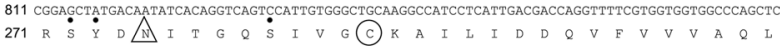
901 TTCGGCGGCTCCCACATT TACAAATACGACGAGAGCTGGACCAAGTTTGTCAAATTCCAAGACATCGAAGTCTCCCGCATTTCCAAGCCC 991 AACGACATCGAGTTGTTTCAGATCGAGGACGAGACGTTCTTCGTCATCGCAGACAGCTCGAAAGCTGGGCTGTCCACAGTGTATAAATGG 331 N D 1081 AACAGCAAAGGATTCTACTCCTACCAGTCCCTGCACGAGTGGTTCAGGGACACGGATGCGGAGTTTGTGGACATAGACGGGAAATCGCAT

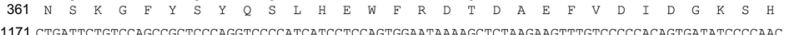

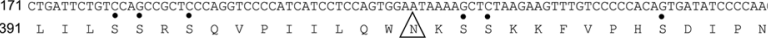
1261 ATGGAGGACGTACTGGCCGTGAAAAGCTTTAGAATGCAGAATGCCCTCTACCTTTCTCTCACCCGCTTCATCGGGGACTCCAGGGTCATG

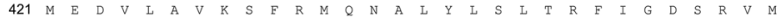
1351 AAGTGGAATAGTAAGGGGTTTGTGGAGATCCAGGCTCTTCCGTCCCGGGGGGCCATGACCCTGCAGCCCTT TTC TTITAAAGATAACCAC P F S E R D N H

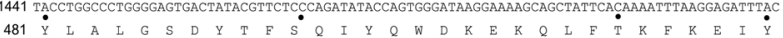
1531 GTGCAAGCGCCTCGTTCCTTCACGGCTGTCTCCACCGACAGGAGAGATTTCTTTTTTGCATCCAGTTTCAAAGGGAAAACAAAGATTTT? 1621 GAaCATATAGTCGTTGACTPAaGTTTGTGA

d 1 MGGAGVVLIL L I A G A G A G V A W R B P K G K (C) P P 91 AGCTGCTCCTGCTCCAAGGATAGTGCCCTGTGTGAGGGCTCTCCAGACCTGCCCGAGAGCTTCTCCCCGACCCTGCTGTCACTCTCACTC 181 GTTAGGACTGGAATCACCCAGCTGAAGGCGGGAAGCTTCCTGAGGGTGCCCACACTGCACCTGCTCCTCTTCACATCCAACTCCTTCTCT $\begin{array}{llllllllllllllllllllllllllllllll}61 & V & R & T & G & I & T & Q & \text { L } & K & A & G & S & F & L & R & V & P & T & L & H & L & L & L & E & T & S & N & S & F & \text { S }\end{array}$

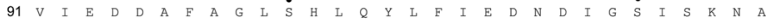
361 CTCAGAGGACTTCGCTCACTCACACACCTGAGCCTGGCCAGTAATAATCTCGAGACCCTCCCCAGATTCCTGTTCCGAGGCCTGGAGACC 121 L $R$ G G L $\quad$ R

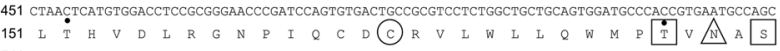
541 GTGGGGACTGGGCCCTGCGCCGGCCCCACTGCTCTGGCCCACATGCAGCTCCGCCACCTGGACCCCAAGACGTTCAAGTGCAGAGCCATA 631 GAGCTGTCCTGGTTCCAGACGGTTCGGGGAGGCGGCGCTGGGCGTGGAGTCCTTCTCCTACCAGGGGGAGCCCCACATGGTCCTGGCACAG

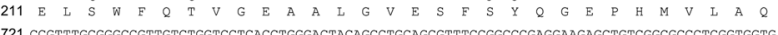
241 P F A G R (C) L V L 811 TCCTGCAAGCCACTGGTGCTGGGCCCGAGCCTCTTCGTGCTGGCCGCCCGCCTGTGGGGTGGCTCGCAGCTGTGGGCCCGGCCCGGCCCC

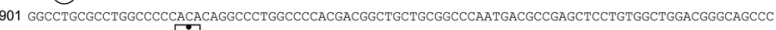
301 G L $\quad$ R L $\quad$ A 991 TGCTTCGTGGTGGCCGACGCCTCCAAAGGGGGGCAGCACCACGCTGCTGTGCCGGGACGGGCCCGGCTTCTACCCTCGCCAGAGCCTGCAC 331 (C) E V V D D A 1081 GCCTGGCACCGGGACACGGATGCCGAGGCCCTCGAGCTGGACGGCCGGCCCCACCTGCT $G C T G G C C T C$ CGCCTCACAGCGGCCCGTGCTC 1171 TTCCACTGGCTCGC

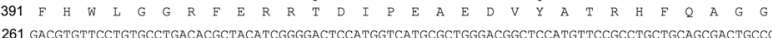

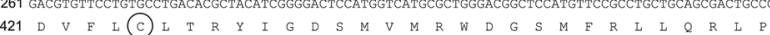
1351 TCGGGTGGTGCCCACGTCTTCCAGCCGCTGCTCATCGCCAGGGACCAGCTGGCTATCCTGGGCAGGGACTTCGCCTTCAGTCAGGTCTTG 451 S $R$ R $G$ A

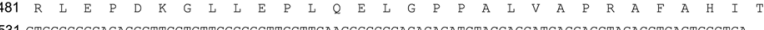

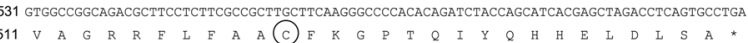




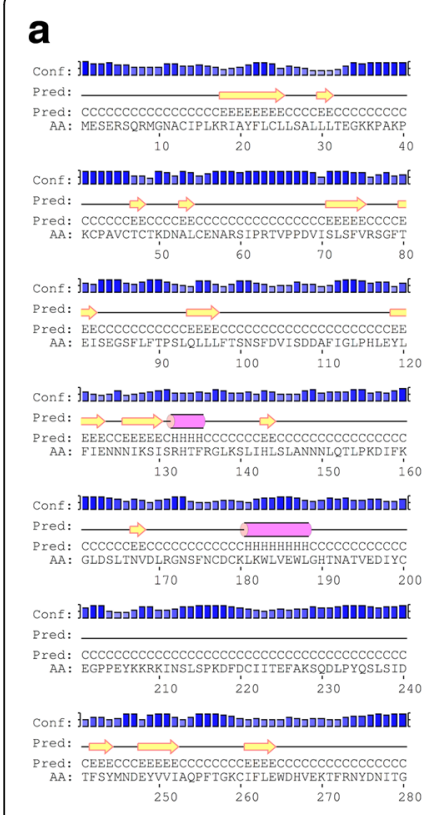

\section{C}

Conf:

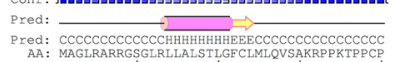

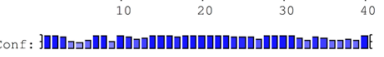

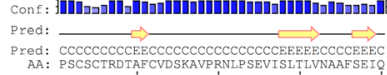

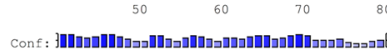

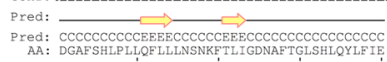

Conf:

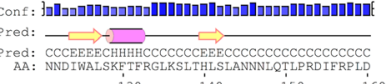

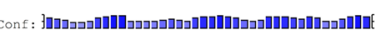

Pred:
$\begin{gathered}\text { Pred: } \\ \text { AA: }\end{gathered}$ CCEECCCCCCCCCCCCHHHHHHACCCCCCCCCCCCCC

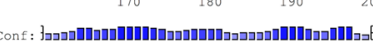

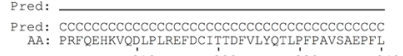

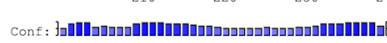

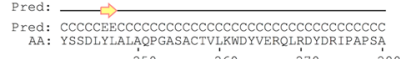

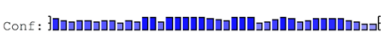

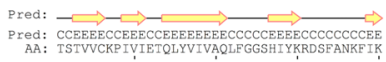

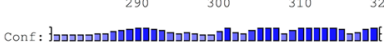

Pred:
Pred:
AA:
ARCCCCCCCCCCCCCCCCCCCCEEEEEECCCCCCCEEE

AA: IQDIEILKIRKPND IETEKIENNWYFVVADSSKAGE TIIY

Conf: \}|

red:
red:
AA: EECCCCCCCCCCCCCCCCCCCEEEEECCCCCCCCCCEE

$\begin{array}{ccc}\text { AA: KWNGNGEYSHQSLHAWYRDTDVEYLETARTPQTLRTPHLT } \\ 370 & 380 & 390\end{array}$

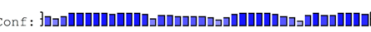

Pred: $\Rightarrow>$
Pred: EECCCCCEEEEECCCCCEECCCCCCCCCCEEEEEEE
AA: LSSSSQRPVIYQNNKATQLIETNQTDIPNMEDYYAVKHF Y

$\begin{array}{ccc}\text { AA: LSSSSQRPVIYYWNKATQLFTNOTD IPNMEDVYAVKHF SY } \\ 410 & 420 & 430\end{array}$

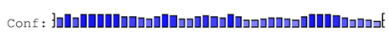

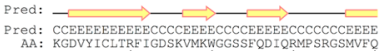

ít

Conf: :

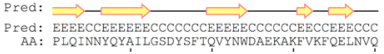

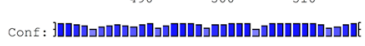

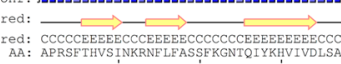

b

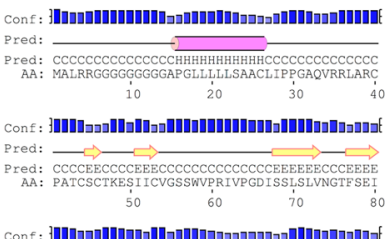

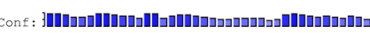

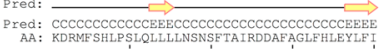

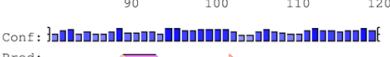

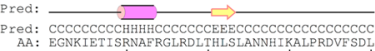

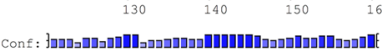

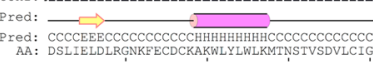

$\begin{array}{ccc}A A: \text { DSLIELDLRGNKFECDCKAKWLYLWLKMTNSTVSDVICIG } \\ 170 & 180 & 190 \quad 200\end{array}$

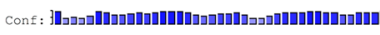

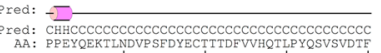
$\dot{210} \quad 220 \quad 230 \quad \dot{2} 0$

Conf: 3mon|l

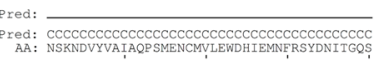

\section{d}

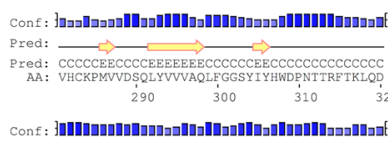

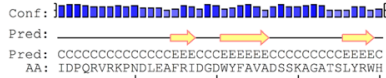

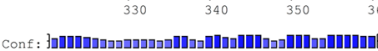

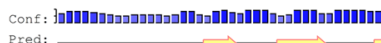

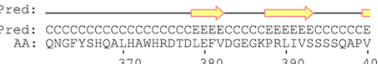

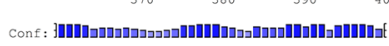

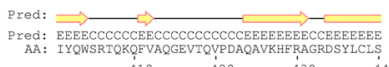

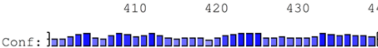

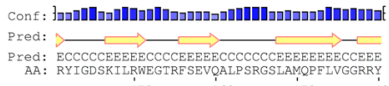

$\begin{array}{lll}450 & 460 \quad 470 \quad 480\end{array}$

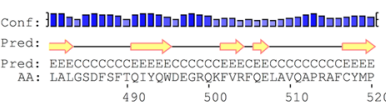

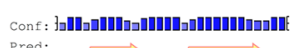

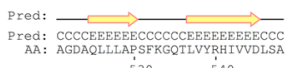

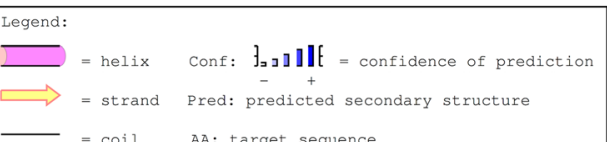

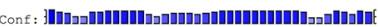

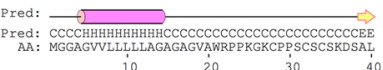

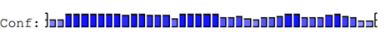

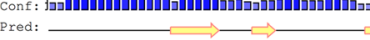

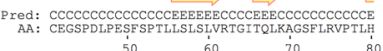

Conf: 3||

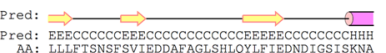

$\begin{array}{llll}90 & 100 & 110 & 120\end{array}$

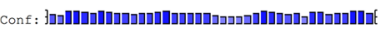

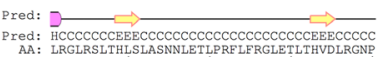

$\begin{array}{llll}130 & 140 & 150 & 160\end{array}$

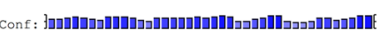

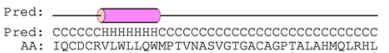

$\begin{array}{lllll}170 & 180 & 190 & 200\end{array}$

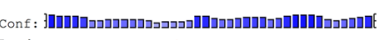

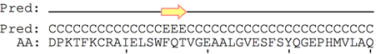

$\begin{array}{llll}210 & 220 & 230 & 240\end{array}$

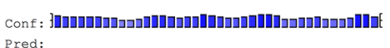

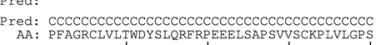

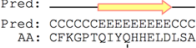

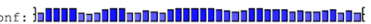

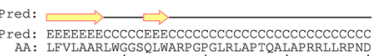

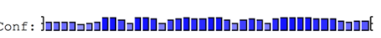

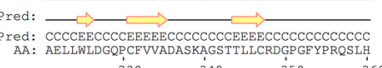

Conf: \}

red: $\longrightarrow$

Pred:

Conf :

red: $\longrightarrow \longrightarrow$

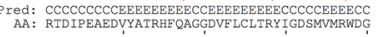

Conf: 3| 3|

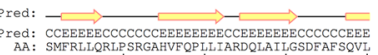

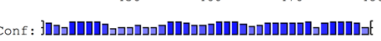

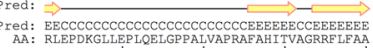

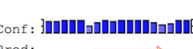

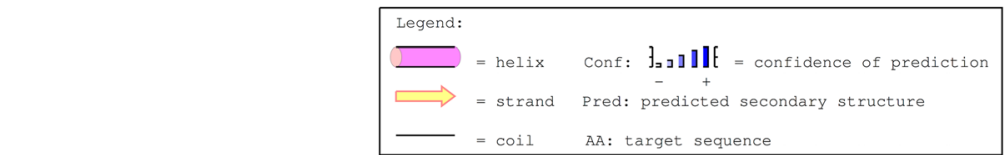

Fig. 2 The predicted secondary structure of the $f L G l$ proteins. Secondary structure of (a) fLGl1, (b) $f L G|2,(\mathbf{c}) f L G| 3$, and (d) fLGl4

three SNPs were found in fLGI1, which included one synonymous SNPs in the coding region and two SNPs in the intron region. Six synonymous SNPs in the coding region and 11 SNPs in the intron and noncoding regions were found in $f L G I 2$. As for $f L G I 3$, one non-synonymous SNP, four synonymous SNPs, and four intronic SNPs were detected. In fLGI4, two non-synonymous SNPs, three synonymous SNPs, and seven intronic SNPs were found. For the three nonsynonymous SNPs found in the $f L G I$ genes, P33A in fLGI3 was heterozygous in a control cat, A17T in fLGI4 was also heterozygous in a control cat, while E49K in fLGI4 was heterozygous in three FSECs. No co-segregation of the disease was found with any 


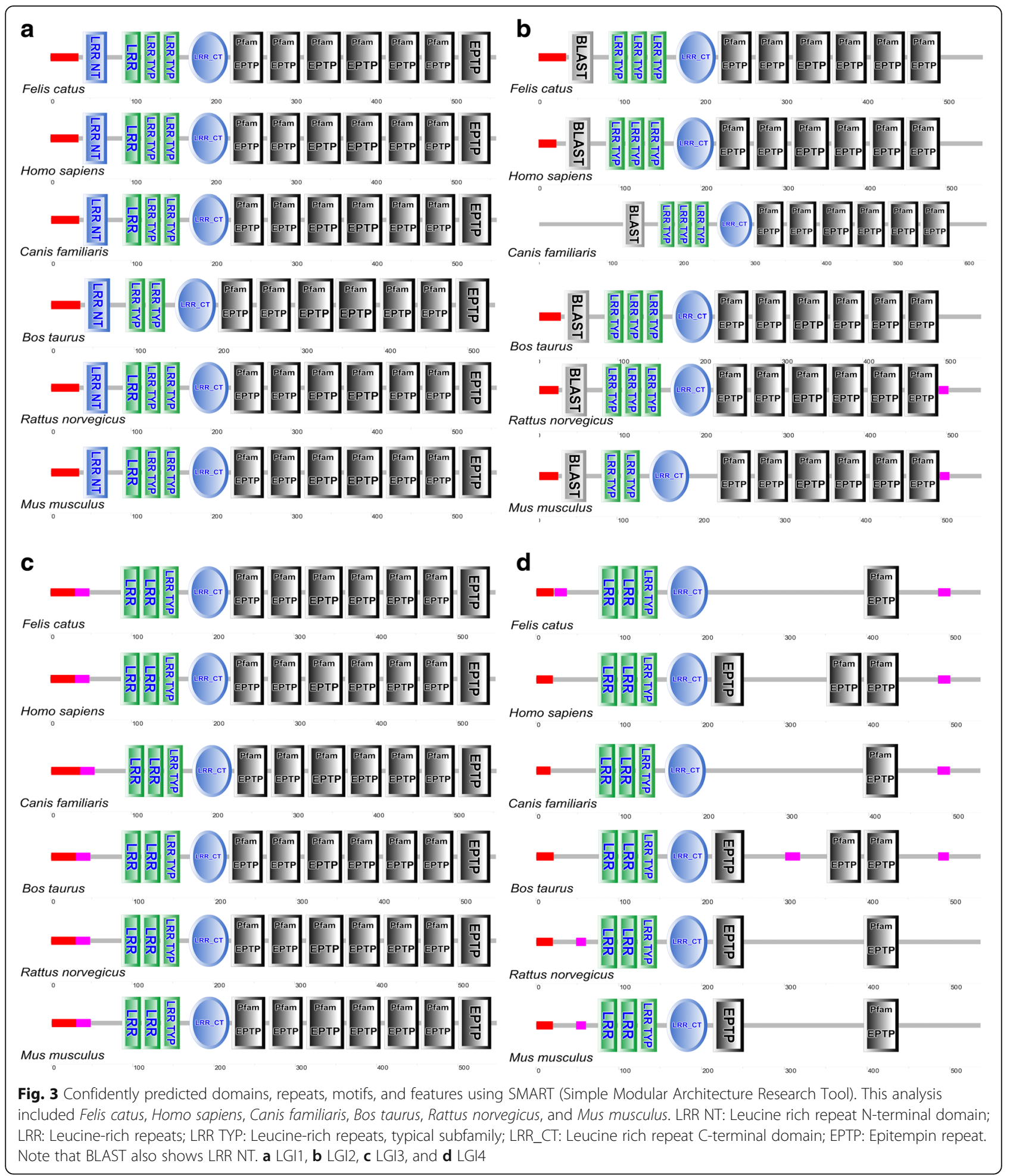

variant. These results are summarized in Table 3 and Additional file 11.

In silico functional analysis for one non-synonymous SNP (P33A) in fLGI3 and two non-synonymous SNPs (A17T, E49K) in fLGI4 were performed using SIFT (Sorting
Intolerant From Tolerant), PolyPhen-2 (Polymorphism Phenotyping v2), and PROVEAN (Protein Variation Effect Analyzer). SIFT predicted P33A in fLGI3 as deleterious, and A17T and E49K in fLGI4 as tolerated (SIFT scores were $0.02,0.26$, and 0.27 , respectively). PP2 
Table 2 Identity of amino acid sequences of the LGI family in other species

\begin{tabular}{lllll}
\hline Species & LGl1 (\%) & LGl2 (\%) & LGl3 (\%) & LGI4 (\%) \\
\hline Homo sapiens & 99.3 & 96.2 & 97.6 & 93.5 \\
Rattus norvegicus & 97.1 & 95.1 & 96.5 & 88.1 \\
Mus musculus & 97.1 & 85.7 & 97.1 & 89.0 \\
Canis familiaris & 100.0 & 96.8 & 96.9 & 96.1 \\
Bos taurus & 95.0 & 96.2 & 96.7 & 92.9 \\
Sus scrofa & 99.1 & 96.6 & 98.4 & 92.8 \\
Equus caballus & 99.5 & 97.5 & 98.0 & 92.9 \\
Gallus gallus & 84.5 & 87.0 & 73.9 & $\mathrm{~N} / \mathrm{A}$ \\
Xenopus tropicalis & 80.7 & 81.6 & $\mathrm{~N} / \mathrm{A}$ & 43.7 \\
All of the above species & 94.7 & 92.7 & 88.5 & 86.3 \\
\hline N/A Not available & & & &
\end{tabular}

predicted P33A in $f L G I 3$ as benign, A17T in $f L G I 4$ as unknown, and E49K in fLGI4 as benign (PP2 scores were 0.002 , not available, and 0.185 , respectively). PROVEAN predicted all SNPs as neutral (PROVEAN scores were $-0.528,-0.346$, and 0.948 , respectively). These results are also summarized in Table 3.

\section{Discussion}

LGI proteins are known to play an important role in synaptic transmission, and their dysfunction may cause neuronal hyperexcitability [22]. Although LGI1 gene mutations can cause autosomal dominant lateral TLE in humans (but not FMTLE), LGI1 conditional knockout mice were reported to have epileptic discharges that started in the hippocampus [35]. Similarly, we previously reported that epileptic seizures in FSECs originated from the hippocampus and/or amygdala [13]. Thus, these data suggest that LGI1 dysfunction may cause seizure onset in the mesial temporal lobe. Mutations of the LGI gene family are also associated with genetic epilepsy in both humans and animals [23-25, 31-33], although the association of LGI3 with disease occurrence remains unclear. Nevertheless, LGI3 was reported to be broadly expressed in the adult mouse brain [34], and to play a regulatory role in neuronal exocytosis [36]. In the present study, we cloned cDNA of the fLGI gene family from feline brain tissue, analyzed their potential functions, and assessed their associations with feline familial epilepsy.

All fLGI family members cloned in this study showed a predicted molecular mass of approximately $60 \mathrm{kDa}$, similar to those in humans [37]. All fLGI family genes were predicted to have a signal peptide followed by a leucinerich repeat domain flanked by cysteine-rich sequences, consistent with the reported previously features in humans [38]. Exonic structural analysis indicated conserved exonic numbers and bps, especially between mammalian species. Further, multiple alignments, homology analysis, and phylogenic tree analysis revealed that the LGI amino acid sequences were conserved with sequences of their analogous in other species, particularly in Homo sapiens, Canis familiaris, Bos taurus, Sus scrofa, and Equus caballus (>90\% homology). LGI1 showed the highest conserved amino acid sequence, suggesting that it may have similar functions to those in other species. Domain analysis also showed conservative structures between mammalian species. Although there were occasional mismatched structures in some mammalian sequences, those sequences were considered not completely accurate. Interaction analysis suggested that the LGI1, 3, and 4 proteins interacted with the a disintegrin and metalloprotease (ADAM) family proteins. ADAM22 and ADAM23 genes were reported to be associated with progressive encephalopathy with epilepsy in human, and with genetic epilepsy in canine, respectively [39-41]. Thus, LGI-ADAM interactions are an area of important focus for future epilepsy research.

In our mutational analysis, we only evaluated exons and exon-intron boundaries, except for exon1 of $f L G I 2$ (we were unable to design the primers because of the high GC content). Thus, we cannot exclude the possibility that haplotypes within genomic regions of fLGI family genes may be associated with FSEC. Additionally, we cannot exclude other types of intronic mutations that could also cause splicing disruptions. Therefore, there is still a possibility that fLGI genes may be causative of epilepsy in FSECs. Furthermore, c.145G > A in fLGI4 which caused E49K was seen in the three affected cats, suggesting this mutation could potentially cause increased risk for epilepsy. We found that FSECs did not have any coding mutations concordant for causing epilepsy in these genes. In veterinary medicine, VGKC/LGI1 complex antibodymediated LE in feline TLE [27], and the LGI2 mutation in benign familial juvenile epilepsy in Lagotto Romagnolo dogs [31], are the only two known diseases associated with LGI genes/proteins. Thus, further studies are required to determine the association of LGI proteins and genes with animal disease. It was reported that rats with a LGI1 missense mutation (L385R) has sound-induced generalized tonic-clonic seizures [42].

Several forms of human familial TLE have been reported, including autosomal dominant and complex inheritance [17-20]. The initial pedigree analysis at the time we identified FSEC, presumed that FSEC might have an autosomal recessive inheritance pattern [7], however, the current extended pedigree also implied the possibilities for other inheritance patterns, such as low-penetrance autosomal dominant or complex inheritance as well. Genetic studies have been performed on multiple human families with FMTLE, and several loci have been mapped in large pedigrees by linkage analysis [43-47]. Most of these loci have not been replicated in other families, 


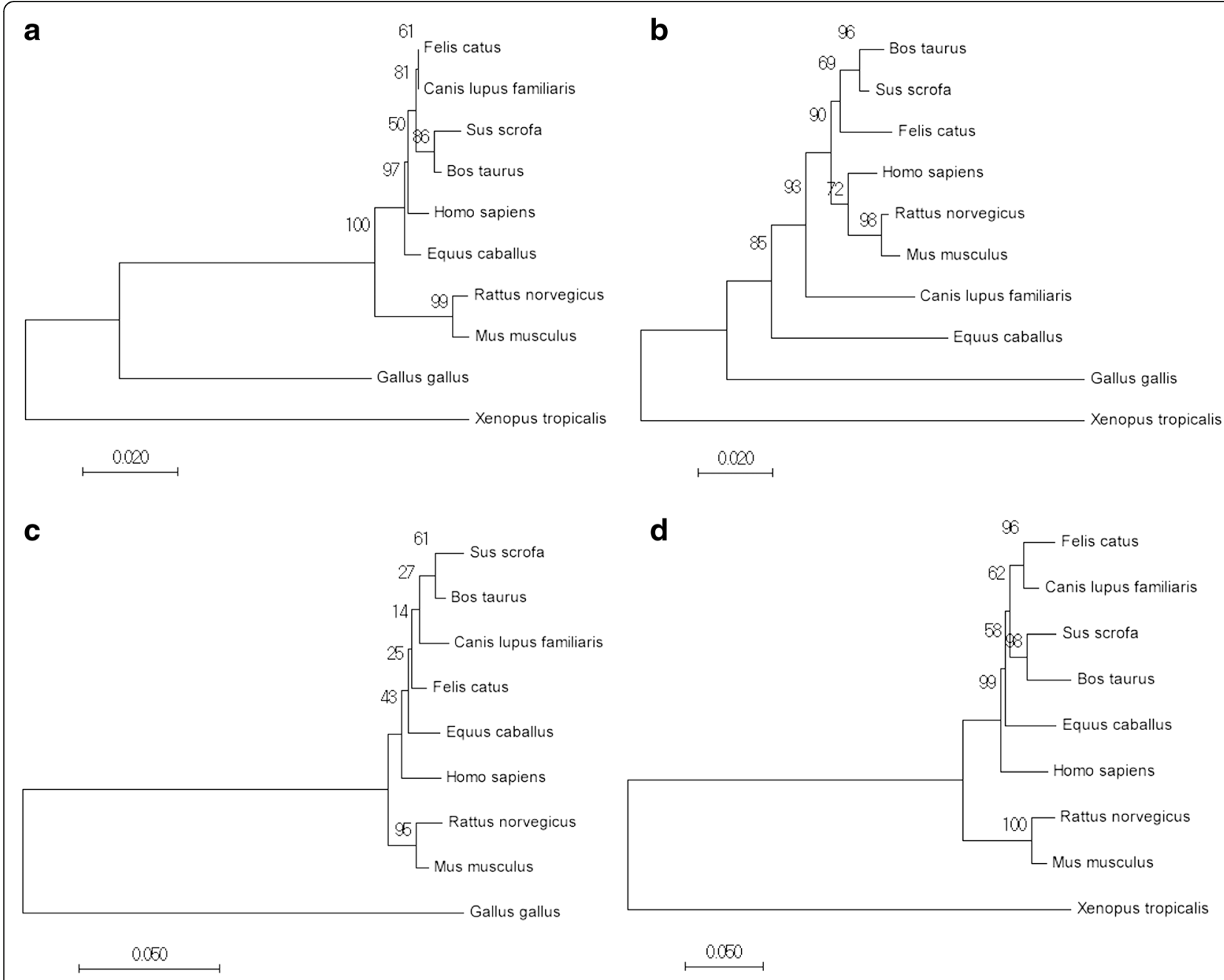

Fig. 4 Phylogenetic trees of the LGl family. The phylogenetic trees of the LGI family proteins were constructed to investigate the evolutionary relationships using the neighbor-joining method with Molecular Evolutionary Genetics Analysis Version (MEGA) 7.0 to calculate the p-distance, 1000 bootstrap replication, and pairwise deletion for the gaps/missing data treatment. Amino acid sequences of the LGI family used for this construction were obtained from GenBank, and their accession numbers are shown in Additional file 10. a LGI1 protein, b LGI2 protein, c LGI3 protein, and $\mathbf{d}$ LGI4 protein. The numbers at the nodes indicate bootstrap majority consensus values from 1000 replicates

Table 3 Allelic and genotypic distribution of non-synonymous mutation found in LGl1-4 in familial spontaneous epileptic cats (FSECs) and controls

\begin{tabular}{|c|c|c|c|c|c|c|c|c|c|c|c|}
\hline \multirow[b]{2}{*}{ LGI3 } & \multirow[t]{2}{*}{ Nucleotide } & \multirow[t]{2}{*}{ Amino Acid } & \multirow[t]{2}{*}{ SIFT prediction } & \multirow[t]{2}{*}{ PP2 prediction } & \multirow[t]{2}{*}{ PROVEAN prediction } & \multirow[t]{2}{*}{ Group (n) } & \multicolumn{3}{|c|}{ Genotype frequencies } & \multicolumn{2}{|c|}{ Allele frequencies } \\
\hline & & & & & & & $\mathrm{C} / \mathrm{C}$ & $C / G$ & $\mathrm{G} / \mathrm{G}$ & $f(C)$ & $f(G)$ \\
\hline & $c .97 C>G$ & p.P33A & Deleteriouss & Benign & Neutral & FSECs(8) & 8 & 0 & 0 & 1 & 0 \\
\hline & & & & & & Controls(8) & 7 & 1 & 0 & 0.9375 & 0.0625 \\
\hline \multirow[t]{6}{*}{ LGl4 } & & & & & & & $\mathrm{G} / \mathrm{G}$ & $\mathrm{G} / \mathrm{A}$ & $\mathrm{A} / \mathrm{A}$ & $f(G)$ & $f(A)$ \\
\hline & C. $49 \mathrm{G}>\mathrm{A}$ & p.A17T & Tolerated & Unknown & Neutral & FSECs(8) & 8 & 0 & 0 & 1 & 0 \\
\hline & & & & & & Controls(8) & 7 & 1 & 0 & 0.9375 & 0.0625 \\
\hline & & & & & & & $\mathrm{G} / \mathrm{G}$ & $\mathrm{G} / \mathrm{A}$ & A/A & $f(G)$ & $f(A)$ \\
\hline & c. $145 G>A$ & p.E49K & Tolerated & Benign & Neutral & FSECs(8) & 5 & 3 & 0 & 0.8125 & 0.1875 \\
\hline & & & & & & Controls(8) & 8 & 0 & 0 & 1 & 0 \\
\hline
\end{tabular}


potentially because of their genetic heterogeneity. Only one of these studies performed whole exome sequencing in addition to linkage analysis, although no putative pathogenic variants were found in the region suggested by linkage analysis [47]. It is possible that whole genome sequencing combined with linkage analysis may reveal the genetic cause of human FMTLE, such as regulatory variants or risk haplotype outside of the coding regions, or genomic rearrangement. Recently, genome-wide analyses including linkage analysis, the transmission disequilibrium test, genome-wide association analysis using the feline SNP array, and whole genome sequencing have become available for feline genetic disorders [48, 49], providing new possibilities for genetic analysis of FSECs.

\section{Conclusion}

In the present study, we cloned, sequenced, and characterized the cDNA sequences of the feline LGI1-4 genes. Using deduced amino acid sequences, we also predicted their physical and chemical properties, performed structural analyses, evaluated the evolutionary association between species, and examined their association with disease in FSECs. We found that the fLGI1-4 amino acid sequences were highly conserved with those of other mammals. However, mutational analysis revealed that the non-synonymous substitutions found in the fLGI family genes were not involved in disease occurrence in FSECs. Further studies using genome-wide strategies, including combinations of linkage analysis, the transmission disequilibrium test, or next generation sequencing, are required to identify the genes involved in epilepsy in FSECs. Nevertheless, our findings provide a further understanding of the functions of the fLGI genes and proteins, and emphasize the presence of FMTLE unrelated to only single mutations within coding regions of LGI family genes.

\section{Methods}

\section{Experimental animals and sample collection}

This study was approved by the Animal Care and Use Committee and the Bioethics Committee of Nippon Veterinary and Life Science University (accession \#26 K-29, 27 K-10, $28 \mathrm{~K}-4$; representative researcher was D.H.). FSECs and control cats were housed in the facility for rearing animal at Nippon Veterinary and Life Science University. Founder cats for the FSEC colony were originally purchased from a commercial laboratory (Narc, Inc., Chiba, Japan; already closed down.) in 2009 and have since been maintained as a colony at Nippon Veterinary and Life Science University [7]. All control cats were obtained from two other commercial laboratories (Shiraishi Laboratory Animals Co., Ltd., Saitama, Japan; already closed down, and Liberty Research, Inc., Waverly, NY, USA). For cDNA cloning, brain tissues were obtained from a normal cat unrelated to the FSEC strain and euthanized previously for another study [29].
After euthanasia, brain tissues (cerebral cortex) were collected within $30 \mathrm{~min}$ and snap frozen in liquid nitrogen. For mutation analysis, eight FSECs (male:female $=6: 2$; median age: 95.5 months [range: $82-180$ months]; median body weight: $3.9 \mathrm{~kg}$ [range: $3.0-5.6 \mathrm{~kg}$ ]) and eight healthy cats without history of seizures, abnormal findings on magnetic resonance imaging or scalp electroencephalography, or relationship with FSECs (male:female $=5: 3$; median age: 73 months [range: $33-74$ months]; median body weight: $3.8 \mathrm{~kg}$ [range: $3.0-6.0 \mathrm{~kg}$ ]) were included.

A $0.5 \mathrm{ml}$ peripheral blood sample was collected by jugular or inner thigh venipuncture without sedation to extract genomic DNA. The median age of the seizure onset in FSECs was 8 months (range: 3-35 months). Six out of eight FSECs showed both seizure forms, one cat showed only spontaneous limbic seizures evolving into generalized epileptic seizures, and the other showed only vestibular stimulation-induced generalized epileptic seizures. All FSECs used in this study had interictal spikes on scalp electroencephalography, which predominantly arose from the temporal region. All cats were monitored by a 24-h video monitoring system for more than 1 year. Spontaneous seizure frequencies were variable among FSECs (range: $0-36 /$ year). Clinical data of FSECs included in this study are summarized in Table 4.

\section{RNA extraction and first strand CDNA synthesis}

Total RNA was extracted from a normal cat brain using Illustra RNA spin columns (GE Healthcare UK Ltd., Little Chalfont, England), according to the manufacturer's instructions. Total RNA was reverse transcribed into first-strand cDNA using ReverTra Ace reverse transcriptase (Toyobo, Osaka, Japan). The cDNA was used as the template to amplify the fLGI1, 2, 3, and 4 genes.

\section{Cloning of full-length fLGI cDNAs}

To amplify overlapping fragments including ORFs of the fLGI1-4 genes, oligonucleotide primers were designed based on the predicted mRNA sequences of the Felis catus LGI1-4 genes (GenBank accession numbers: XM_003994222.3, XM_011281979.1, XM_003984713.3, and XM_003997904.3, respectively) using Primer3.0 online software (http://www.bioinformatics.nl/cgi-bin/ primer3plus/primer3plus.cgi/). PCR was performed with AmpliTaq Gold 360 Master Mix (Thermo Fisher Scientific, Waltham, MA, USA) using brain cDNA as a template. PCR cycle conditions were follows: $95{ }^{\circ} \mathrm{C}$ for $10 \mathrm{~min}$, followed by 38 cycles of denaturation $\left(95^{\circ} \mathrm{C}\right.$ for $\left.30 \mathrm{~s}\right)$, annealing (30 s), and extension $\left(72{ }^{\circ} \mathrm{C}\right.$ for $\left.60 \mathrm{~s}\right)$, followed by a final extension $\left(72{ }^{\circ} \mathrm{C}\right.$ for $\left.7 \mathrm{~min}\right)$. The set of primers used and each annealing temperature are summarized in Additional file 12. PCR products were detected by $2 \%$ agarose gel electrophoresis, isolated from the agarose, 
Table 4 Clinical summary of FSECs included in this study

\begin{tabular}{|c|c|c|c|c|c|c|c|c|}
\hline FSECS & $\begin{array}{l}\text { Age } \\
\text { (months) }\end{array}$ & Sex & $\begin{array}{l}\text { Seizure } \\
\text { phenotype }\end{array}$ & $\begin{array}{l}\text { Seizure dominant } \\
\text { form }\end{array}$ & $\begin{array}{l}\text { Spontaneous seizure } \\
\text { frequency (/year) }\end{array}$ & $\begin{array}{l}\text { Age at Onset } \\
\text { (months) }\end{array}$ & Father & Mother \\
\hline$\# 1$ & 84 & M & SS & SS & About 36 & 8 & Asymptomatic* & Asymptomatic \\
\hline$\# 2$ & 74 & M & SS\&VSS & SS & About 24 & 8 & Asymptomatic & Asymptomatic \\
\hline \#3 & 61 & $F$ & SS\&VSS & SS & $0-2$ & 10 & Asymptomatic & Asymptomatic \\
\hline \#4 & 67 & M & SS\&VSS & VSS & About 2 & 17 & $\begin{array}{l}\text { Symptomatic } \\
(\# 1)\end{array}$ & $\begin{array}{l}\text { Symptomatic } \\
(\# 6)\end{array}$ \\
\hline \#5 & 129 & M & SS\&VSS & SS & $0-4$ & 36 & Asymptomatic & Asymptomatic \\
\hline \#6 & 87 & $\mathrm{~F}$ & SS\&VSS & VSS & $0-2$ & 8 & Asymptomatic* & Asymptomatic \\
\hline \#7 & 74 & M & SS\&VSS & VSS & $0-1$ & 35 & Symptomatic & Symptomatic $\neq \mathbb{9}$ \\
\hline \#8 & 79 & M & VSS & VSS & N/A & 4 & Asymptomatic & Symptomatic $\neq \boldsymbol{q}$ \\
\hline
\end{tabular}

${ }^{*},+,{ }^{\ddagger}$ Cats with the same symbol are the same individual. "Cats showed spontaneous seizures (SS) only. Vestibular-stimulated seizures (VSS) were rare in all cats used in this study. $M$ Male. $F$ Female

and purified using the Wizard SV Gel and PCR Clean-Up System (Promega, Fitchburg, WI, USA).

For TA cloning, purified cDNA were ligated with T4 DNA ligase and cloned into the pGEM-T Easy Vector System (Promega). The vector containing purified PCR product was transformed into competent high $\mathrm{DH} 5 \alpha$ cells (Toyobo). Plasmids were isolated from DH5 $\alpha$ cells using the NucleoSpin Plasmid QuickPure Kit (Takara Bio, Shiga, Japan). As the $5^{\prime}$-region of the coding sequence of fLGI2 was not represented in the predicted sequences (GenBank accession numbers: XM_011281979.1), 3'- and $5^{\prime}$-rapid amplification of cDNA ends PCR was performed. The sequences at the $5^{\prime}$-end of LGI2 were amplified using the SMARTer RACE 5'/3' Kit (Takara Bio), following the manufacturer's instructions.
DNA extraction from peripheral blood, PCR for mutation detection, and product purification

Genomic DNA from eight FSECs and eight control cats was extracted from peripheral blood collected in tubes containing ethylenediaminetetraacetic acid using the QIAamp DNA Mini Kit (Qiagen, Hilden, Germany), according to the manufacturer's instructions. PCR was performed as described above for cloning. Primers (flanking the intron/exon boundaries) were designed based on the predicted genomic sequences for each of the fLGI1-4 genes (NC_018733.2, NC_018726.2, NC_018726.2, and NC_018737.2, respectively) using Primer 3.0 online software. Detection and purification of PCR products was performed as described above for LGI gene cloning, except that the concentration of agarose gel used was $1.8 \%$.

Table $\mathbf{5}$ List of bioinformatics analysis software used in this study

\begin{tabular}{lll}
\hline Bioinformatics analysis software & URL & Prediction purpose \\
\hline ORF finder & https://www.ncbi.n/m.nih.gov/orffinder/ & Predict ORF \\
ProtParam & http://web.expasy.org/protparam/ & Basic properties \\
PsiPred & http://bioinf.cs.ucl.ac.uk/psipred/ & Secondary structures \\
NetOGlyc 4.0 & http://www.cbs.dtu.dk/services/NetOGlyc/ & O-glycosylation sites \\
NetNGlyc 1.0 & http://www.cbs.dtu.dk/services/NetNGlyc/ & N-glycosylation sites \\
NetPhos 3.1 & http://www.cbs.dtu.dk/services/NetPhos/ & Phosphorylation sites \\
TMHMM Server v. 2.0 & http://www.cbs.dtu.dk/services/TMHMM/ & Transmembrane domains \\
SignalP 4.1 & http://www.cbs.dtu.dk/services/SignalP/ & Signal peptides \\
SCRATCH & http://scratch.proteomics.ics.uci.edu/ & Disulfide bonds \\
PSORT II & https://psort.hgc.jp/form2.html & Sub cellular distribution \\
LALIGN & http://www.ch.embnet.org/software/LALIGN_form.html & Pairwise alignments \\
SMART & http://smart.embl.de/ & Structural domains \\
STRING 10.0 & http://string-db.org/ & Proteins interaction \\
SIFT & http://sift.bii.a-star.edu.sg/ & Predicting pathogenicity \\
PP2 & http://genetics.bwh.harvard.edu/pph2/ & Predicting pathogenicity \\
PROVEAN & http://provean.jcvi.org & Predicting pathogenicity \\
\hline
\end{tabular}


Primer sequences and annealing temperatures are shown in Additional file 13.

\section{Sequencing}

Purified DNA and purified PCR products were sequenced using an Applied Biosystems 3130xl Genetic Analyzer (Applied Biosystems, Foster City, CA, USA) or 3730xl DNA Analyzer (Applied Biosystems) using BigDye Terminator v3.1 (Applied Biosystems). Sequencing results were analyzed using the CLC sequence viewer (Version 7.0; CLC Bio, Waltham, MA, USA). For mutation analysis, direct sequencing of the PCR products was performed twice.

\section{Bioinformatics analyses of $\mathrm{fLGl}$ genes}

The full-length cDNA sequences of the fLGI genes were assembled using CLC Sequence Viewer. The basic bioinformatics analysis software used in this study are shown in Table 5. The amino acid sequences were deduced from our cDNA sequences. The genomic sequences of LGI1-4 genes from other species (accession numbers shown in Additional file 5) were used to perform exonic sequence structure analysis. All amino acid sequences used for multiple sequence alignment and homology analysis are summarized in Additional file 10. Pairwise alignments between the Felis catus and other species were performed with LALIGN, using the following parameters: scoring matrix (BLOSUM50), gap open/extension penalty $(-12 /-2)$. Multiple sequence alignments were constructed using DNAMAN version 9.122 (Trial version; Lynnon Biosoft, San Ramon, CA, USA). A phylogenetic analysis was performed using the neighbor-joining methods (bootstrap phylogeny test, 1000 replicates) with MEGA 7.0 software to calculate the p-distance. In silico functional analysis of non-synonymous mutations was performed using SIFT, PP2 and PROVEAN. In SIFT analysis, the amino acid substitution are predicted damaging if the score is $\leq 0.05$, and tolerated if the score is $>0.05$. PP2 score ranges from 0.0 (tolerated) to 1.0 (deleterious). PROVEAN was used with a threshold value of -2.5 .

\section{Additional files}

Additional file 1: The subcellular distribution of feline leucine-rich glioma-inactivated (fLGI) proteins. The subcellular distribution of fLGI proteins are represented in pie charts. (PDF $301 \mathrm{~kb}$ )

Additional file 2: Confidently predicted domains, repeats, and motifs of LG proteins by SMART (Simple Modular Architecture Research Tool) (DOCX $41 \mathrm{~kb}$ )

Additional file 3: The confidence views of the LGl protein interaction. Stronger associations are represented by thicker lines. (a) Confidence views of $f L G l 1$ protein interactions, (b) fLGI2 protein interactions, (c) fLGI3 protein interactions, and (d) fLGI4 protein interactions (TIFF $674 \mathrm{~kb}$ )

Additional file 4: Exonic structures of the coding sequences of the LGl family genes from Felis catus and other species. (a-d) show comparison of exonic structures between species for the LGI1-LGI4 genes, respectively. Amino acid sequences and genomic sequences are provided in Additional file 5.
Colored blocks represent exons. Gray blocks represent introns. Numbers over the colored blocks indicate base pairs that construct exons (TIFF $15627 \mathrm{~kb}$ )

Additional file $\mathbf{5}$ Accession numbers of the amino acid sequences and genomic sequences used for exonic structural analysis (DOCX $84 \mathrm{~kb}$ )

Additional file 6 Multiple alignment of LGl1 amino acid sequences from Felis catus and other species. Multiple alignment was constructed using DNAMAN 9.122. The black, red, and light blue shading represent 100\% conserved, less-conserved $(\geq 75 \%)$, and non-conserved $(\geq 50 \%)$ amino acids, respectively (JPEG $14409 \mathrm{~kb}$ )

Additional file $\mathbf{7}$ Multiple alignment of LGI2 amino acid sequences from Felis catus and other species. Multiple alignment was constructed using DNAMAN 9.122. The black, red, and light blue shading represent 100\% conserved, less-conserved $(\geq 75 \%)$, and non-conserved ( $\geq 50 \%)$ amino acids, respectively (JPEG $14074 \mathrm{~kb}$ )

Additional file 8 Multiple alignment of LGI3 amino acid sequences from Felis catus and other species. Multiple alignment was constructed using DNAMAN 9.122. The black, red, and light blue shading represent 100\% conserved, less-conserved $(\geq 75 \%)$, and non-conserved $(\geq 50 \%)$ amino acids, respectively (JPEG $13184 \mathrm{~kb}$ )

Additional file 9 Multiple alignment of $\mathrm{LGI} 4$ amino acid sequences from Felis catus and other species. Multiple alignment was constructed using DNAMAN 9.122. The black, red, and light blue shading represent 100\% conserved, less-conserved $(\geq 75 \%)$, and non-conserved $(\geq 50 \%)$ amino acids, respectively (JPEG $11862 \mathrm{~kb}$ )

Additional file $\mathbf{1 0}$ List of amino acid sequences of LGI proteins used in the analysis (DOCX $59 \mathrm{~kb}$ )

Additional file 11 Allelic and genotypic distribution of synonymous and intronic polymorphisms other than non-synonymous mutation found in LGl1-4 genes in familial spontaneous epileptic cats (FPSCs) and controls (DOCX $86 \mathrm{~kb})$

Additional file 12 List of oligonucleotide primers used for molecular cloning of ORFs of $\mathrm{fLGl}$ genes. In rapid-amplification of CDNA ends (RACE), touchdown PCR was performed, with annealing temperatures of $70{ }^{\circ} \mathrm{C}$ for five cycles and $68{ }^{\circ} \mathrm{C}$ for 20 cycles (DOCX $85 \mathrm{~kb}$ )

Additional file 13 List of oligonucleotide primers used in sequencing and mutation analysis of the fLGl1-4 genes (DOCX $108 \mathrm{~kb}$ )

\section{Abbreviations}

ADAM: A disintegrin and metalloprotease; ADLTE: Autosomal dominant lateral temporal lobe epilepsy; Bp: Base pair(s); cDNA: DNA complementary to RNA; EPTP: Epitempin; FEPSO: Feline complex partial seizure with orofacial involvement; fLGI: Feline leucine-rich glioma inactivated; FMTLE: Familial mesial temporal lobe epilepsy; FSEC: Familial spontaneous epileptic cats; LE: Limbic encephalitis; LGI: Leucine-rich glioma inactivated; LRR: Leucine rich repeat; ORF: Open reading frame; RACE: Rapid-amplification of CDNA ends; RT-PCR: Reverse transcription-polymerase chain reaction; SNPs: Single nucleotide polymorphisms; TLE: Temporal lobe epilepsy; VGKC: Voltage-gated K Channel

\section{Acknowledgements}

We thank Dr. Hirotaka Igarashi for critical review of this manuscript.

\section{Funding}

This study was partially supported by a Grant of the Science Research Promotion Fund from the Promotion and Mutual Corporation for Private School of Japan (2015-2017 [representative researcher: DH]). The funders had no role in the design of the study and experiments, analysis, and the interpretation of data and in writing the manuscript.

\section{Availability of data and materials}

All data and materials can be found in the tables, figures, and additional files.

\section{Authors' contributions}

DH and YY conceived and designed the study. YY and AFI performed the experiments and analyzed the data. $\mathrm{DH}, \mathrm{YY}, \mathrm{YH}, \mathrm{SM}$, and TK coordinated the clinical evaluation of the studied animals and the collection of samples. YY drafted the manuscript. YY, DH, AFI, and MF finalized the manuscript. All authors read and approved the final manuscript. 


\section{Ethics approval}

This study was approved by the Animal Care and Use Committee and the Bioethics Committee of Nippon Veterinary and Life Science University (accession \#26 K-29, 27 K-10, 28 K-4; representative researcher was D.H.).

\section{Consent for publication}

Not applicable.

\section{Competing interests}

The authors declare that they have no competing interests.

\section{Publisher's Note}

Springer Nature remains neutral with regard to jurisdictional claims in published maps and institutional affiliations.

Received: 26 July 2017 Accepted: 28 November 2017

Published online: 13 December 2017

\section{References}

1. Hauser WA. Incidence and prevalence. In: Engel J, Pedley TA, editors. Epilepsy: a comprehensive textbook. Philadelphia: Lippincott-Raven; 1997. p. 47-59.

2. Hardies K, Weckhuysen S, De Jonghe P, Suls A. Lessons learned from gene identification studies in Mendelian epilepsy disorders. Eur J Hum Genet. 2016;24:961-7.

3. EpiPM Consortium. A roadmap for precision medicine in the epilepsies. Lancet Neurol. 2015:14:1219-28.

4. Walker LE, Mirza N, Yip VL, Marson AG, Pirmohamed M. Personalized medicine approaches in epilepsy. J Intern Med. 2015;277:218-34.

5. Reif PS, Tsai MH, Helbig I, Rosenow F, Klein KM. Precision medicine in genetic epilepsies: break of dawn? Expert Rev Neurother. 2017;17:381-92.

6. Yokoi N, Fukata Y, Kase D, Miyazaki T, Jaegle M, Ohkawa T, Takahashi N, Iwanari H, Mochizuki Y, Hamakubo T, Imoto K, Meijer D, Watanabe M, Fukata M. Chemical corrector treatment ameliorates increased seizure susceptibility in a mouse model of familial epilepsy. Nat Med. 2015;21:19-26.

7. Kuwabara T, Hasegawa D, Ogawa F, Kobayashi M, Fujita M, Suzuki H, Matsuki N, Orima H. A familial spontaneous epileptic feline strain: a novel model of idiopathic/genetic epilepsy. Epilepsy Res. 2010:92:85-8.

8. Kitz S, Thalhammer JG, Glantschnigg U, Wrzosek M, Klang A, Halasz P, Shouse MN, Pakozdy A. Feline temporal lobe epilepsy: review of the experimental literature. J Vet Intern Med. 2017;31:633-40

9. Cizinauskas S, Fatzer R, Schenkel M, et al. Can idiopathic epilepsy be confirmed in cats? J Vet Intern Med. 2003, 246;17 abstract

10. Pakozdy A, Sarchahi AA, Leschnik M, Tichy AG, Halasz P, Thalhammer JG. Treatment and long-term follow-up of cats with suspected primary epilepsy. J Feline Med Surg. 2013;15:267-73.

11. Wahle AM, Brühschwein A, Matiasek K, Putschbach K, Wagner E, Mueller RS, Fischer A. Clinical characterization of epilepsy of unknown cause in cats. J Vet Intern Med. 2014;28:182-8.

12. King JT Jr, LaMotte CC. El mouse as a model of focal epilepsy: a review. Epilepsia 1989;30:257-265.

13. Hasegawa D, Mizoguchi S, Kuwabara T, Hamamoto Y, Ogawa F, Matsuki N, Uchida K, Fujita M. Electroencephalographic features of familial spontaneous epileptic cats. Epilepsy Res. 2014;108:1018-25.

14. Mizoguchi S, Hasegawa D, Kuwabara T, Hamamoto Y, Ogawa F, Fujiwara A, Matsuki N, Fujita M. Magnetic resonance volumetry of the hippocampus in familial spontaneous epileptic cats. Epilepsy Res. 2014;108:1940-4.

15. Mizoguchi S, Hasegawa D, Hamamoto Y, Yu Y, Kuwabara T, Fujiwara-lgarashi A, Fujita M. Interictal diffusion and perfusion magnetic resonance imaging features of cats with familial spontaneous epilepsy. Am J Vet Res. 2017;78:305-10.

16. Hamamoto $Y$, Hasegawa D, Mizoguchi S, Yu Y, Wada M, Kuwabara T, Fujiwara-Igarashi A, Fujita M. Changes in the interictal and early postictal diffusion and perfusion magnetic resonance parameters in familial spontaneous epileptic cats. Epilepsy Res. 2017;133:76-82.

17. Berkovic SF, McIntosh A, Howell RA, Mitchell A, Sheffield LJ, Hopper JL. Familial temporal lobe epilepsy: a common disorder identified in twins. Ann Neurol. 1996:40:227-35.

18. Baulac S, Gourfinkel-An I, Nabbout R, Huberfeld G, Serratosa J, Leguern E, Baulac M. Fever, genes, and epilepsy. Lancet Neurol. 2004;3:421-30.

19. Striano P, Gambardella A, Coppola A, Di Bonaventura C, Bovo G, Diani E, Boaretto F, Egeo G, Ciampa C, Labate A, Testoni S, Passarelli D, Manna I, Sferro C, Aguglia U, Caranci F, Giallonardo AT, Striano S, Nobile C,
Michelucci R. Familial mesial temporal lobe epilepsy (FMTLE) : a clinical and genetic study of 15 Italian families. J Neurol. 2008;255:16-23.

20. Crompton DE, Scheffer IE, Taylor I, Cook MJ, McKelvie PA, Vears DF, Lawrence KM, McMahon JM, Grinton BE, McIntosh AM, Berkovic SF. Familial mesial temporal lobe epilepsy: a benign epilepsy syndrome showing complex inheritance. Brain. 2010;133:3221-31.

21. Kegel L, Aunin E, Meijer D, Bermingham JR. LGl proteins in the nervous system. ASN Neuro. 2013;5:167-81.

22. Pakozdy A, Patzl M, Zimmermann L, Jokinen TS, Glantschnigg U, Kelemen A, Hasegawa D. LGI Proteins and epilepsy in human and animals. J Vet Intern Med. 2015;29:997-1005.

23. Gu W, Wevers A, Schröder H, Grzeschik KH, Derst C, Brodtkorb E, de Vos R, Steinlein OK. The LGI1 gene involved in lateral temporal lobe epilepsy belongs to a new subfamily of leucine-rich repeat proteins. FEBS Lett. 2002;519:71-6.

24. Kalachikov S, Evgrafov O, Ross B, Winawer M, Barker-Cummings C, Martinelli Boneschi F, Choi C, Morozov P, Das K, Teplitskaya E, Yu A, Cayanis E, Penchaszadeh G, Kottmann AH, Pedley TA, Hauser WA, Ottman R, Gilliam TC. Mutations in LGI1 cause autosomal-dominant partial epilepsy with auditory features. Nat Genet. 2002;30:335-41.

25. Morante-Redolat JM, Gorostidi-Pagola A, Piquer-Sirerol S, Sáenz A, Poza JJ, Galán J, Gesk S, Sarafidou T, Mautner VF, Binelli S, Staub E, Hinzmann B, French L, Prud'homme JF, Passarelli D, Scannapieco P, Tassinari CA, Avanzini G, Martí-Massó JF, Kluwe L, Deloukas P, Moschonas NK, Michelucci R, Siebert R, Nobile C, Pérez-Tur J, López de Munain A. Mutations in the LGl1/ Epitempin gene on 10q24 cause autosomal dominant lateral temporal epilepsy. Hum Mol Genet. 2002;11:1119-28.

26. Pakozdy A, Gruber A, Kneiss S, Leschnik M, Halasz P, Thalhammer JG. Complex partial cluster seizures in cats with orofacial involvement. J Feline Med Surg. 2011;13:687-93.

27. Pakozdy A, Halasz P, Klang A, Bauer J, Leschnik M, Tichy A, Thalhammer JG, Lang B, Vincent A. Suspected limbic encephalitis and seizure in cats associated with voltage-gated potassium channel (VGKC) complex antibody. J Vet Intern Med. 2013;27:212-4.

28. Pakozdy A, Glantschnigg U, Leschnik M, Hechinger H, Moloney T, Lang B, Halasz $P$, Vincent A. EEG-confirmed epileptic activity in a cat with VGKC-complex/LGI1 antibody-associated limbic encephalitis. Epileptic Disord. 2014;16:116-20.

29. Yu Y, Hasegawa D, Hamamoto Y, Mizoguchi S, Kuwabara T, Fujiwara-lgarashi A, Tsuboi M, Chambers JK, Fujita M, Uchida K. Neuropathological features of the hippocampus and amygdala in familial spontaneous epileptic cats. Am J Vet Res. In Press

30. Miller TD, Chong TT, Aimola Davies AM, Ng TW, Johnson MR, Irani SR, Vincent A, Husain M, Jacob S, Maddison P, Kennard C, Gowland PA, Rosenthal CR. Focal CA3 hippocampal subfield atrophy following LGI1 VGKC-complex antibody limbic encephalitis. Brain. 2017;140:1212-9.

31. Seppälä EH, Jokinen TS, Fukata M, Fukata Y, Webster MT, Karlsson EK, Kilpinen SK, Steffen F, Dietschi E, Leeb T, Eklund R, Zhao X, Rilstone JJ, Lindblad-Toh K, Minassian BA, Lohi H. LGI2 truncation causes a remitting focal epilepsy in dogs. PLoS Genet. 2011;7:e1002194.

32. Gu W, Sander T, Becker T, Steinlein OK Genotypic association of exonic LG|4 polymorphisms and childhood absence epilepsy. Neurogenetics. 2004;5:41-4.

33. Ishii A, Zhang B, Kaneko S, Hirose S. Positive association between benign familial infantile convulsions and LGI4. Brain Dev. 2010;32:538-43.

34. Lee SE, Lee AY, Park WJ, Jun DH, Kwon NS, Baek KJ, Kim YG, Yun HY. Mouse LGI3 gene: expression in brain and promoter analysis. Gene. 2006;372:8-17.

35. Boillot M, Huneau C, Marsan E, Lehongre K, Navarro V, Ishida S, Dufresnois B, Ozkaynak E, Garrigue J, Miles R, Martin B, Leguern E, Anderson MP, Baulac S. Glutamatergic neuron-targeted loss of LGl1 epilepsy gene results in seizures. Brain. 2014;137:2984-96.

36. Park WJ, Lee SE, Kwon NS, Baek KJ, Kim DS, Yun HY. Leucine-rich glioma inactivated 3 associates with syntaxin 1. Neurosci Lett. 2008:444:240-4.

37. Chernova OB, Somerville RP, Cowell JK. A novel gene, LGl1, from 10q24 is rearranged and downregulated in malignant brain tumors. Oncogene. 1998 17:2873-81.

38. Kobe B, Kajava AV. The leucine-rich repeat as a protein recognition motif. Curr Opin Struct Biol. 2001;11:725-32.

39. Koskinen LL, Seppälä EH, Belanger JM, Arumilli M, Hakosalo O, Jokinen P, Nevalainen EM, Viitmaa R, Jokinen TS, Oberbauer AM, Lohi H. Identification of a common risk haplotype for canine idiopathic epilepsy in the ADAM23 gene. BMC Genomics. 2015;16:465.

40. Muona M, Fukata Y, Anttonen AK, Laari A, Palotie A, Pihko $H$, Lönnqvist $T$, Valanne L, Somer M, Fukata M, Lehesjoki AE. Dysfunctional ADAM22 
implicated in progressive encephalopathy with cortical atrophy and epilepsy. Neurol Genet. 2016;2:e46.

41. Koskinen LL, Seppälä EH, Weissl J, Jokinen TS, Viitmaa R, Hänninen RL, Quignon P, Fischer A, André C, Lohi H. ADAM23 is a common risk gene for canine idiopathic epilepsy. BMC Genet. 2017;18:8.

42. Baulac S, Ishida S, Mashimo T, Boillot M, Fumoto N, Kuwamura M, Ohno Y, Takizawa A, Aoto T, Ueda M, Ikeda A, LeGuern E, Takahashi R, Serikawa T. A rat model for LGI1-related epilepsies. Hum Mol Genet. 2012;21:3546-57.

43. Baulac S, Picard F, Herman A, Feingold J, Genin E, Hirsch E, Prud'homme JF, Baulac M, Brice A, LeGuern E. Evidence for digenic inheritance in a family with both febrile convulsions and temporal lobe epilepsy implicating chromosomes 18qter and 1q25-q31. Ann Neurol. 2001;49:786-92.

44. Claes L, Audenaert D, Deprez L, Van Paesschen W, Depondt C, Goossens D, Del-Favero J, Van Broeckhoven C, De Jonghe P. Novel locus on chromosome 12q22-q23.3 responsible for familial temporal lobe epilepsy associated with febrile seizures. J Med Genet. 2004;41:710-4.

45. Hedera P, Blair MA, Andermann E, Andermann F, D'Agostino D, Taylor KA, Chahine L, Pandolfo M, Bradford Y, Haines $J$, Abou-Khalil B. Familial mesial temporal lobe epilepsy maps to chromosome 4q13.2-q21.3. Neurology. 2007:68:2107-12.

46. Chahine L, Abou-Khalil B, Siren A, Andermann F, Hedera P, Ge Q, Andermann E, Pandolfo M. A new locus for familial temporal lobe epilepsy on chromosome 3q. Epilepsy Res. 2013;106:338-44.

47. Fanciulli M, Di Bonaventura C, Egeo G, Fattouch J, Dazzo E, Radovic S, Spadotto A, Giallonardo AT, Nobile C. Suggestive linkage of familial mesial temporal lobe epilepsy to chromosome 3q26. Epilepsy Res. 2014;108:232-40.

48. Alhaddad H, Gandolfi B, Grahn RA, Rah HC, Peterson CB, Maggs DJ, Good $\mathrm{KL}$, Pedersen NC, Lyons LA. Genome-wide association and linkage analyses localize a progressive retinal atrophy locus in Persian cats. Mamm Genome. 2014;25:354-62

49. Lyons LA, Creighton EK, Alhaddad H, Beale HC, Grahn RA, Rah H, Maggs DJ, Helps CR, Gandolfi B. Whole genome sequencing in cats, identifies new models for blindness in AIPL1 and somite segmentation in HES7. BMC Genomics. 2016;17:265.

\section{Submit your next manuscript to BioMed Central and we will help you at every step:}

- We accept pre-submission inquiries

- Our selector tool helps you to find the most relevant journal

- We provide round the clock customer support

- Convenient online submission

- Thorough peer review

- Inclusion in PubMed and all major indexing services

- Maximum visibility for your research

Submit your manuscript at www.biomedcentral.com/submit

) Biomed Central 